\title{
Induction of endoplasmic reticulum calcium pump expression during early leukemic $B$ cell differentiation
}

Lamia Ait Ghezali ${ }^{1,2}$, Atousa Arbabian³ ${ }^{3}$, Hervé Roudot ${ }^{1,2,5}$, Jean-Philippe Brouland ${ }^{4}$, Fanny Baran-Marszak 1,2,5, Evelyn Salvaris ${ }^{6}$, Andrew Boyd ${ }^{7}$, Hans G. Drexler ${ }^{8}$, Agnes Enyedi ${ }^{9}$, Remi Letestu ${ }^{1,2,5}$, Nadine Varin-Blank ${ }^{1,2}$ and Bela Papp ${ }^{1,2,10^{*}}$ (1)

\begin{abstract}
Background: Endoplasmic reticulum (ER) calcium storage and release play important roles in B lymphocyte maturation, survival, antigen-dependent cell activation and immunoglobulin synthesis. Calcium is accumulated in the endoplasmic reticulum (ER) by Sarco/Endoplasmic Reticulum Calcium ATPases (SERCA enzymes). Because lymphocyte function is critically dependent on SERCA activity, it is important to understand qualitative and quantitative changes of SERCA protein expression that occur during B lymphoid differentiation and leukemogenesis.

Methods: In this work we investigated the modulation of SERCA expression during the pharmacologically induced differentiation of leukemic precursor B lymphoblast cell lines that carry the E2A-PBX1 fusion oncoprotein. Changes of SERCA levels during differentiation were determined and compared to those of established early B lymphoid differentiation markers. SERCA expression of the cells was compared to that of mature B cell lines as well, and the effect of the direct inhibition of SERCA-dependent calcium transport on the differentiation process was investigated.

Results: We show that E2A-PBX1+ leukemia cells simultaneously express SERCA2 and SERCA3-type calcium pumps; however, their SERCA3 expression is markedly inferior to that of mature B cells. Activation of protein kinase $C$ enzymes by phorbol ester leads to phenotypic differentiation of the cells, and this is accompanied by the induction of SERCA3 expression. Direct pharmacological inhibition of SERCA-dependent calcium transport during phorbol ester treatment interferes with the differentiation process.

Conclusion: These data show that the calcium pump composition of the ER is concurrent with increased SERCA3 expression during the differentiation of precursor B acute lymphoblastic leukemia cells, that a cross-talk exists between SERCA function and the control of differentiation, and that SERCA3 may constitute an interesting new marker for the study of early B cell phenotype.
\end{abstract}

Keywords: Pre-B acute lymphoblastic leukemia, Phorbol ester, Endoplasmic reticulum, SERCA, Calcium transport

\footnotetext{
*Correspondence: belapapp2@yahoo.fr; bela.papp@inserm.fr

${ }^{1}$ Institut National de la Santé et de la Recherche Médicale, U978 Bobigny,

France

2Université Paris-13, PRES Sorbonne Paris-Cité, 74, rue Marcel Cachin 93017,

Bobigny, France

Full list of author information is available at the end of the article
} 


\section{Background}

Calcium signaling is essential for B lymphocyte responses. The activation of the B cell antigen receptor and other important receptors leads, via PLC- $\gamma$ activation and subsequent IP3 production, to the release of calcium accumulated in the ER via IP3 receptor calcium channels. ER calcium release, coupled to capacitative calcium influx from the extracellular space through Orai-type calcium channels leads to increased cytosolic calcium levels, the activation of calcineurin and various PKC isoforms and the activation of NF-KB and NFAT-type transcription factors that orchestrate $\mathrm{B}$ cell activation [1-10].

Calcium-dependent activation is critically dependent on the function of Sarco/Endoplasmic Reticulum Calcium ATPases (SERCA enzymes). Located in the ER membrane, SERCA proteins accumulate calcium ions from the cytosol into the ER lumen by ATP-dependent active transport, creating a several thousand fold calcium concentration gradient from the ER (high micromolar) towards the cytosol (low nanomolar) [11-14]. SERCA activity is therefore a prerequisite for IP3-induced calcium release to occur $[15,16]$, and, by recapturing calcium during a calcium release event, SERCA enzymes also shape the amplitude and the duration of calcium signals [17], and thus modulate cell activation [18]. Mature B cells express simultaneously SERCA2b and SERCA3-type calcium pumps $[19,20]$. The calcium affinity of SERCA3 is markedly weaker $\left(\mathrm{K}_{\mathrm{Ca}}^{2+} \sim 1.2 \mu \mathrm{M}\right)$ than that of SERCA2b $\left(\mathrm{K}_{\mathrm{Ca}}^{2+} \sim 0.2 \mu \mathrm{M}\right)[13,21]$. Due to its lower calcium affinity, SERCA3 will tolerate higher calcium rises during ER calcium release [13, 21-24]. The SERCA2b/SERCA3 protein ratio constitutes a unique mechanism whereby the avidity of the ER for calcium can be modulated in a cell $[25,26]$.

Early B cell differentiation proceeds through several consecutive steps during which an initially immature lymphoid precursor acquires the cellular machinery required for the function of the differentiated $\mathrm{B}$ cell. Phenotypic changes that occur during this process, such as the loss or gain of various markers and gene expression regulatory networks, have already been described in considerable detail [27-29]. However, little is known so far about the functional remodeling of ER calcium homeostasis and about changes of SERCA-dependent ER calcium sequestration that may occur during early $B$ lymphoid differentiation.

Precursor-B ALL cells are blocked at early stages of Bcell differentiation due to the presence of various genetic lesions that give rise to different fusion oncoproteins such as E2A-PBX1 (TCF3-PBX1), BCR-ABL1, MLL-ENL (KMT2A-MLLT1), TEL-AML1 (ETV6-RUNX1) or others. These oncoproteins activate distinct molecular oncogenic mechanisms, influence prognosis, and are widely used for the molecular classification of precursor-B ALL [30, 31].
In order to investigate SERCA expression during early B cell differentiation, and to gain insight into its defects in leukemia, we investigated the effects of protein kinase $C$ activation by a phorbol ester on the phenotype and SERCA expression of various precursor B ALL cell lines in vitro. Phorbol esters activate PKC [32], and are known to induce differentiation of several myeloid leukemia cell types accompanied by increased SERCA3 expression [33, 34]. We show that the differentiation blockage of E2A-PBX1expressing precursor B-ALL cells can be abolished in vitro by the pharmacological activation of PKC, and that the differentiation of these cells is accompanied by marked changes in SERCA expression. Whereas untreated cells express simultaneously SERCA2 and SERCA3-type calcium pumps, the expression of SERCA3, a lower calcium affinity isoenzyme $[13,21,24]$ is preferentially induced upon differentiation, indicating that ER calcium accumulation is functionally remodeled during this process. In addition, we show that the direct pharmacological inhibition of SERCAdependent calcium transport interferes with the differentiation process as detected by CD20 expression.

\section{Methods}

\section{Cell culture}

Cell lines used in this study were obtained from DSMZ (Braunschweig, Germany), ATCC (Manassas, VA), ECACC (Porton Down, UK) or from the originators (see Additional file 1: Table S1). Authenticity check of cell lines was performed by short tandem repeat analysis by the commercial providers. Early passage cells were cultured in suspension in the appropriate culture medium at $37{ }^{\circ} \mathrm{C}$ in humidified cell culture incubators in a $95 \%$ air $5 \% \mathrm{CO}_{2}$ atmosphere. Fetal calf serum that supported vigorous growth of the Kasumi-2 line as single cells without clumping was selected for culture. Mycoplasma screening was performed by polymerase chain reaction using the G238 mycoplasma detection kit of Applied Biological Materials, Euromedex, Souffelweyersheim, France. Cell densities and viabilities of exponentially growing cultures were determined by cell counting using a Malassez chamber and trypan blue exclusion.

\section{Reagents and treatments}

Before treatments cells were centrifuged and suspended in new complete medium at densities indicated in Figures and distributed into $60 \mathrm{~cm}^{2}$ plastic Petri dishes. Non-adherent dishes were used for the various cell lines because treatment in adherent conditions resulted in the adherence of a minor fraction $(<5 \%)$ of the cells following treatments, for example for Kasumi- 2 cells. Treatment of MHH-CALL3 cells was done in cell culture grade adherent Petri dishes, since treatment of this cell line leads to the adherence of the overwhelming majority $(>90 \%)$ of viable cells. 
PMA, Gö 6983 and GF 109203X, thapsigargin, cyclopiazonic acid and 2,5-di(tert)butyl-1,4-benzohidroquinone were obtained from Sigma-Aldrich France, SaintQuentin Fallavier, France. Drugs were added to cells from concentrated stock solutions made in dimethylsulfoxide (DMSO). DMSO vehicle did not exceed $0.01 \%$, was included in controls and did not interfere with the experiments.

When PMA treatments were applied in the presence of Gö 6983 or GF 109203X, these were added to the cell cultures 30 min before the addition of PMA. PMA and thapsigargin in double treatment experiments were added simultaneously. Drugs were thereafter present during the entire experiment.

\section{Western blotting and flow cytometry}

After the appropriate treatments, cells were harvested by centrifugation and washed with ice cold $150 \mathrm{mM} \mathrm{NaCl}$. Total cellular protein was then precipitated with 5\% trichloroacetic acid (TCA), centrifuged and dissolved in modified SDS PAGE lysis buffer. This sample preparation process, as well as quantification of the protein content of lysates was performed as described in [35]. Equal amounts of total cellular protein $(60 \mu \mathrm{g} /$ well $)$ were applied to polyacrylamide gels, and SDS PAGE followed by immunoblotting onto nitrocellulose was done as previously described [35]. After electrotransfer, identical protein loading was checked by Ponceau red staining and densitometry [35].

Western blotting for SERCA2 and SERCA3 was performed using the IID8 (Sigma-Aldrich, $0.4 \mu \mathrm{g} / \mathrm{ml}$ ) and the PLIM430 mouse monoclonal (10x diluted hybridoma cell culture supernatant) antibodies, respectively, and peroxidase-conjugated anti-mouse Ig antibodies as in [35]. Luminescent signal obtained with the ECL Prime (Amersham) and SuperSignal West Femto Maximum Sensitivity (Thermo Fisher) reagents was detected, quantified and analyzed using a BioRad ChemiDoc MP image acquisition system and the Image Lab 4.0 software. Immunoblot signal intensities were normalized to the corresponding total protein values as obtained by Ponceau red staining, and are expressed as fold change in treated cells versus untreated control.

Detection by Western blotting of CD20 (clone L26 purified mouse monoclonal anti-human CD20cy, Dako Denmark A/S, $0.2 \mu \mathrm{g} / \mathrm{ml}$ ), RAG-1 (Santa Cruz Biotechnology, sc-5599, H-300, rabbit polyclonal IgG $0.2 \mu \mathrm{g} /$ $\mathrm{ml}$ ), TdT (clone EPR2976Y, rabbit hybridoma culture supernatant monoclonal antibody, dilution:3500x, Epitomics), CD19 (clone LE-CD19 purified mouse monoclonal anti-human CD19, Thermo Fisher Scientific, $0.33 \mu \mathrm{g} / \mathrm{ml}$ ) was performed similarly.

Detection and analysis of expression of various lymphoid phenotypic markers (CD3, CD5, CD10, CD19,
CD20, CD22, CD34, CD38, CD45, FMC7, TdT, $\mathrm{\kappa}$ and $\lambda$ light chains and IgM) by flow cytometry was done as previously described [36, 37].

\section{Cytology and immunocytochemistry}

Immunocytochemical staining for CD20 expression was performed on cytologic smears. Suspensions of treated and untreated control cells of packed cell volume ratio of approximately $50 \%$ were applied to poly-lysine coated microscopic slides and air-dried overnight. Following fixation in acetone at room temperature for $10 \mathrm{~min}$ and drying the slides were rehydrated and labeled for CD20 expression using the Clone L26 monoclonal mouse antiCD20 antibody (Dakocytomation, Les Ulis, France) at a concentration of $6 \mu \mathrm{g} / \mathrm{ml}$ in Dako REAL ${ }^{\mathrm{TM}}$ antibody diluent (Dakocytomation), using an indirect avidin-biotinperoxidase method with 3,3'diaminobenzidine (DAB) as chromogen on an automated immunostainer (Benchmark ${ }^{\circ}$, Ventana Medical Systems, Illkirch, France). Endogenous peroxidase activity was blocked by treatment with $3 \%$ hydrogen peroxide in phosphate-buffered saline for 10 min. Incubation with the CD20-specific antibody was carried out at $37^{\circ} \mathrm{C}$ for $30 \mathrm{~min}$, and labeling was revealed using the Ventana $i \mathrm{View}^{\circ} \mathrm{DAB}$ Detection kit with copper enhancement, according to the instructions of the manufacturer. Slides were counterstained with hematoxylin and bluing reagent (Ventana). Cells were photographed using a Zeiss Axio Scope.A1 microscope and an AxioCam ICc1 camera using the Axiovision 4.8.2 software. May-Grünwald-Giemsa staining of cytological smears was done using standard methods.

\section{Real time PCR}

Following treatments cells were centrifuged, and RNA was extracted with the RNeasy Mini Kit of Qiagen (Courtaboeuf, France). RT-PCR was performed on $1 \mu \mathrm{g}$ of total RNA using the Iscript cDNA synthesis kit of Bio-Rad (Marne la Coquette, France) TaqMan gene expression assays were performed on an ABI prism 7000 apparatus (Applied Biosystems, Courtaboeuf, France) with the Hs 99999905_m1, Hs 0054487_m1 and Hs 01024558_m1 primer pairs (Applied Biosystems, Life Technologies, Fisher Scientific, Illkirch, France) for GAPDH, ATP2A2 (SERCA2) and ATP2A3 (SERCA3), respectively. Relative gene expression levels after treatments were calculated by the $2^{-\Delta \Delta \mathrm{Ct}}$ method normalized to GAPDH, and expressed as fold change compared to untreated cells [38].

\section{Statistical analysis}

Data are presented as the mean \pm SEM, and correspond to at least three independent experiments. Statistical analysis was performed using Student's $t$ test with GraphPad Prism. 


\section{Results}

Induction of SERCA expression in precursor B ALL cells As investigated in the Kasumi-2 and RCH-ACV cell lines that carry the $\mathrm{t}(1 ; 19)$ (q23;p13) translocation and express the E2A-PBX1 fusion oncoprotein, PMA treatment led to enhanced SERCA3 expression. This could be observed from $10^{-10}-10^{-9} \mathrm{M}$ PMA, and reached a plateau in the $10^{-8}-10^{-7}$ concentration range (Fig. 1a and b). Induction of SERCA3 expression was also manifested on the mRNA level. As shown in Fig. 1c and d, induction of SERCA3 mRNA expression was observed in both Kasumi-2 and RCH-ACV cells, already at $12 \mathrm{~h}$ of treatments, and this followed a reproducible biphasic pattern with a 5-6-fold enhancement when compared to untreated control. Moreover, the moderate enhancement of
SERCA2 protein expression observed in Kasumi-2 cells could also be observed on the mRNA level.

Significant induction of SERCA3 protein expression was observed starting at day 1-2 and reached a plateau at day 4-5 (Fig. 2a and b). Maximal induction in the cell lines was approximately five- to sevenfold when compared to untreated cells. DMSO vehicle when applied alone was without effect. As shown in Fig. 2c and d, PMA treatment in Kasumi-2 cells led to inhibition of cell proliferation (Panel C) with maintained viability (Panel D) up till day 5, whereas longer treatments led to significant cell death.

SERCA3 expression was induced by PMA in all available precursor B ALL cell lines bearing the $t(1 ; 19) / E 2 A-$ PBX1 translocation. As shown in Fig. 3, the Kasumi-2,

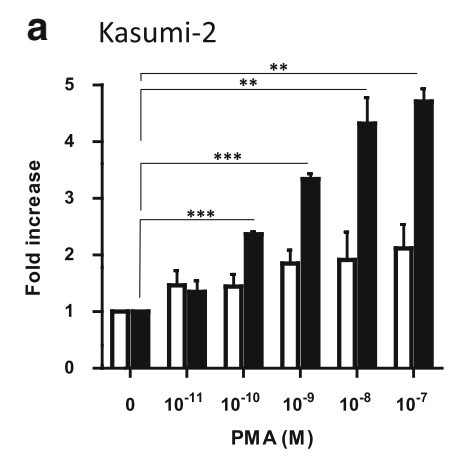

b RCH-ACV
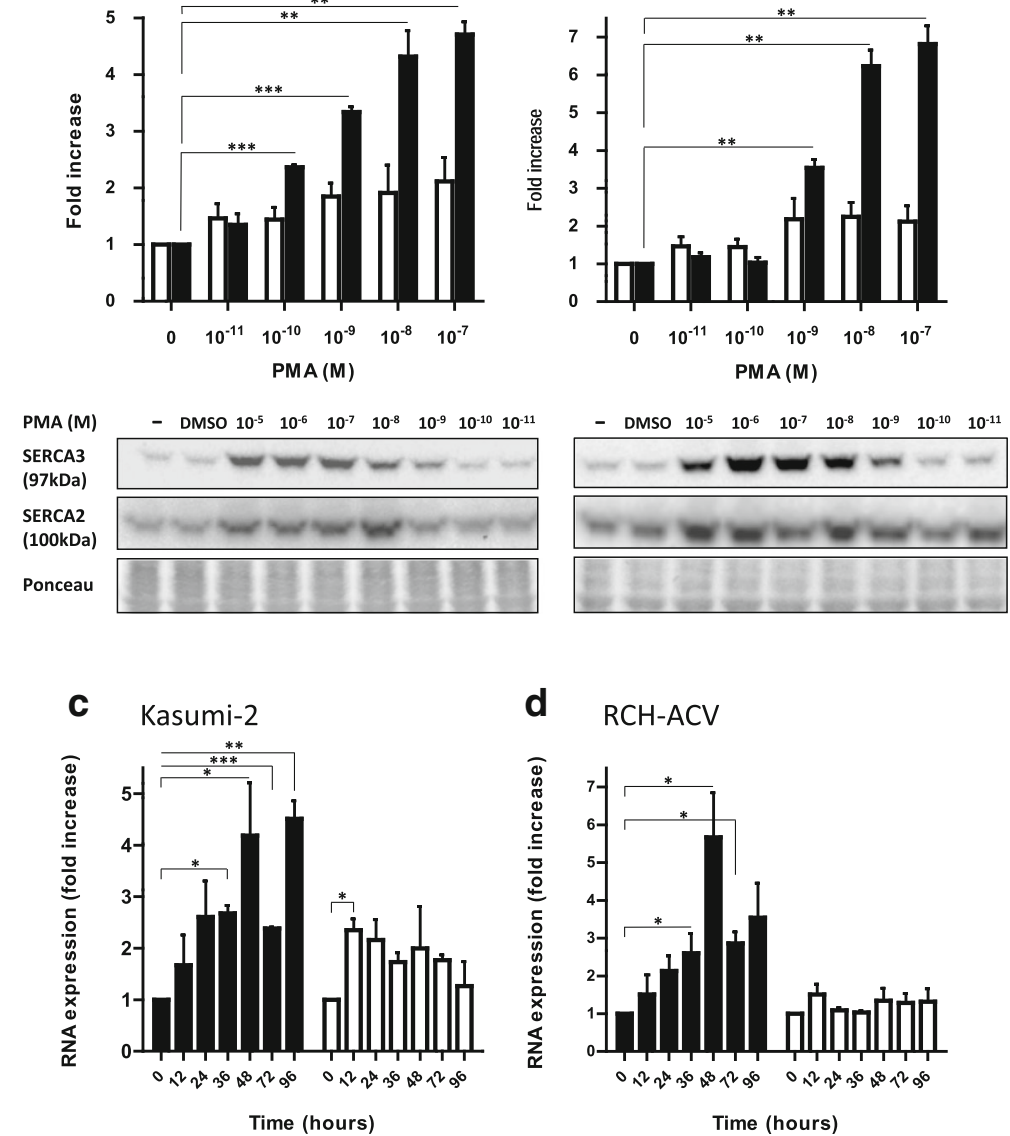

Fig. 1 Induction of SERCA3 expression in precursor B ALL cell lines. Kasumi-2 (a) and RCH-ACV (b) cells were treated by various concentrations of PMA for 5 days, and SERCA3 (closed columns; 97 kDa) as well as SERCA2 (open columns; $100 \mathrm{kDa}$ ) expression was detected by Western immunoblotting (lower part) and quantified (upper part). Equal total cellular protein loading of blots was checked by Ponceau red staining. PMA treatment led to an approximately five- or sevenfold increase of SERCA3 expression in Kasumi-2 and RCH-ACV cells, respectively. Concentration-dependent induction of SERCA expression by PMA could be observed from $10^{-10}-10^{-9} \mathrm{M}$ and reached a plateau around $10^{-8}-10^{-7} \mathrm{M}$, whereas DMSO vehicle was without effect. c and $\mathbf{d}$ : Kasumi-2 and RCH-ACV cells were treated with $10^{-8}$ M PMA, and SERCA3 (closed columns), as well as SERCA2 mRNA levels (open columns) were measured by quantitative RT-PCR and normalized to GAPDH as described in Methods. When compared to untreated cells, SERCA3 mRNA expression peaked at day 2 of treatment, and a rebound could be observed at day 4 

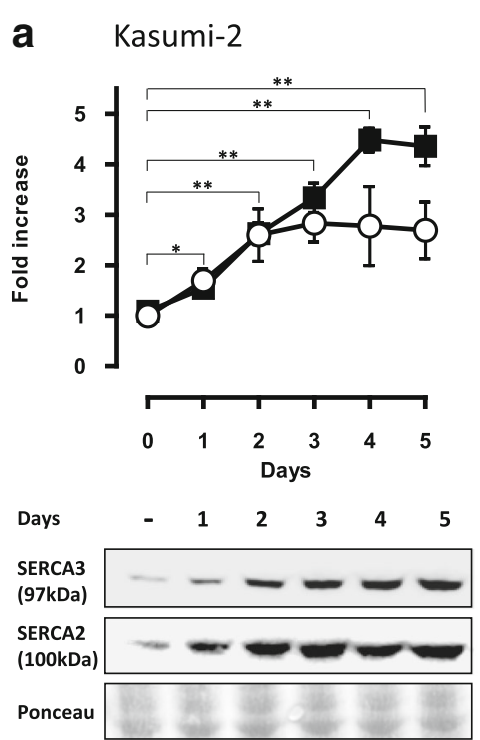

C

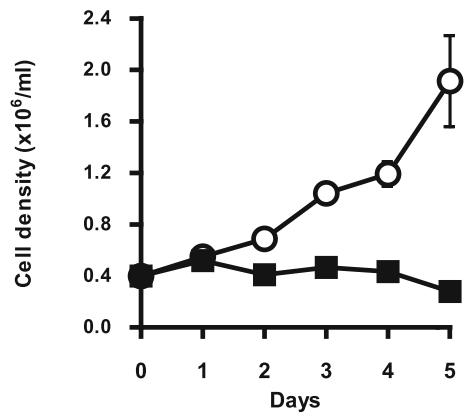

b $\mathrm{RCH}-\mathrm{ACV}$
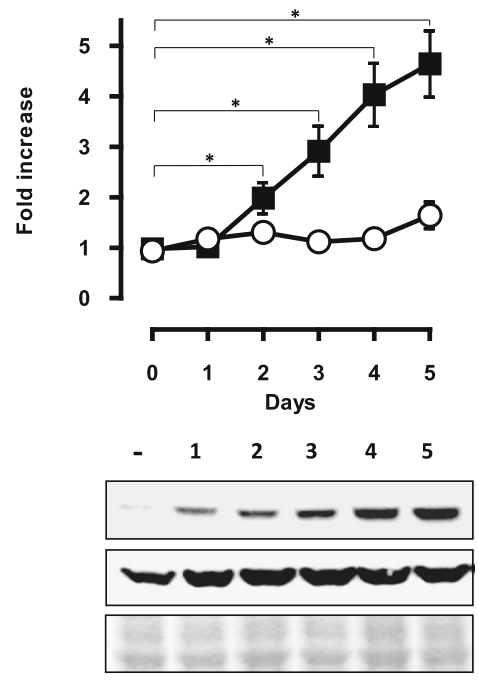

d

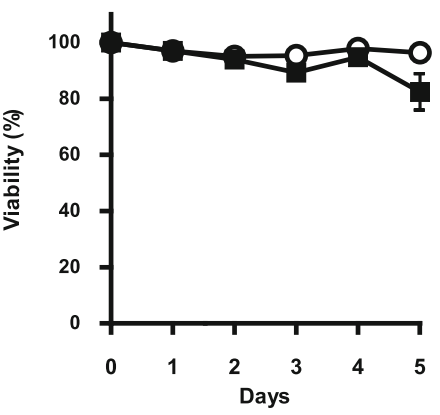

Fig. 2 Time course of SERCA expression in PMA-treated precursor B ALL cell lines. Kasumi-2 (a) and RCH-ACV (b) cells were treated with $10^{-7} \mathrm{M}$ PMA and SERCA3 (closed squares) as well as SERCA2 (open circles) expression was detected by Western blotting (lower part) and quantified (upper part). Equal protein loading was checked by Ponceau red staining of the membranes. Induction of SERCA3 expression was detected from days 1-2 and reached a plateau after 4-5 days. Treatment (Kasumi-2 cells, PMA: $10^{-8} \mathrm{M}$ ) led to rapid growth arrest (c) with maintained viability (d) during 5 days

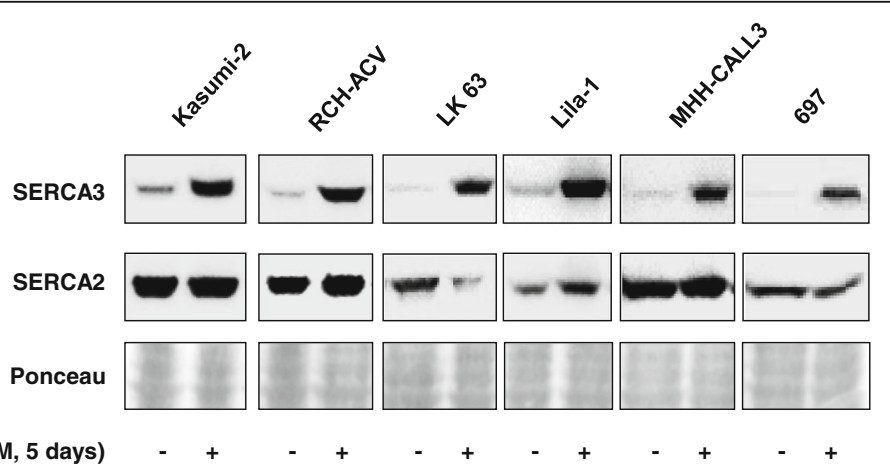

Fig. 3 SERCA protein expression in various precursor B ALL cell lines. Induction of SERCA3 expression by PMA. Semi-quantitative Western blotting analysis of SERCA2 and SERCA3 levels in equal amounts of total cellular protein lysates obtained from untreated (-) and PMA-treated (+) cells. PMA treatment led to enhanced SERCA3 expression in all t(1;19)/E2A-PBX $1^{+}$cell lines 
RCH-ACV, LK63, Lila-1, MHH-CALL3 and 697 cell lines all simultaneously expressed SERCA2 and SERCA3 calcium pump isoforms. When the cells were treated with $10^{-7}$ M PMA for 5 days, the expression of SERCA3 protein was strongly enhanced in all cell lines, whereas expression of the SERCA2 protein remained unchanged or was slightly modified in a cell-line dependent manner. This indicates that the induction of SERCA3 expression is a universal phenomenon in all E2A-PBX1 expressing cell lines investigated in this study. On the other hand, we found that PMA treatment of precursor B ALL cell lines (Nalm-21, TOM-1, BV173, KOPN-8 and Reh) of other molecular types (see Additional file 1: Table S1) led to the death of cultures within 24-48 h.

\section{PMA treatment leads to cell differentiation}

The phenotype of Kasumi-2 precursor B ALL cells was investigated before and after stimulation with $10^{-7} \mathrm{M}$ PMA for 5 days using established markers of early B cell differentiation. Whereas before treatment cells expressed high levels of TdT and RAG-1 and low levels of CD20 proteins, PMA treatment led to strong CD20 expression and to the simultaneous down-regulation of $\mathrm{TdT}$ and RAG-1 expression (Fig. 4a).

PMA-treatment of cells led to the formation of multicellular aggregates and to morphological changes similar to a plasmacytoid morphology (Fig. 4b). Whereas untreated cells were round, small and displayed high nucleocytoplasmic ratio and round nuclei, treatment with $10^{-8}$ M PMA for 4 days led to increased cell size, more abundant, often excentral cytoplasm, lower nucleocytoplasmic ratio and frequently bilobed nuclei with clear paranuclear areas.

As studied by flow cytometry, and similarly to previous observations made on the LK63 and Lila-1 $\mathrm{t}(1 ; 19)$ cell lines [39], untreated Kasumi-2 cells displayed a precursor B lymphoid phenotype $\left(\mathrm{CD}^{-}, \mathrm{CD} 5^{-}, \mathrm{CD} 10^{+}, \mathrm{CD} 19^{+}\right.$, $\left.\mathrm{CD} 20^{+/-}, \mathrm{CD} 22^{+}, \mathrm{CD} 34^{-}, \mathrm{CD} 38^{+}, \mathrm{CD} 45^{+}, \mathrm{TdT}^{+}\right) . \mathrm{As}$

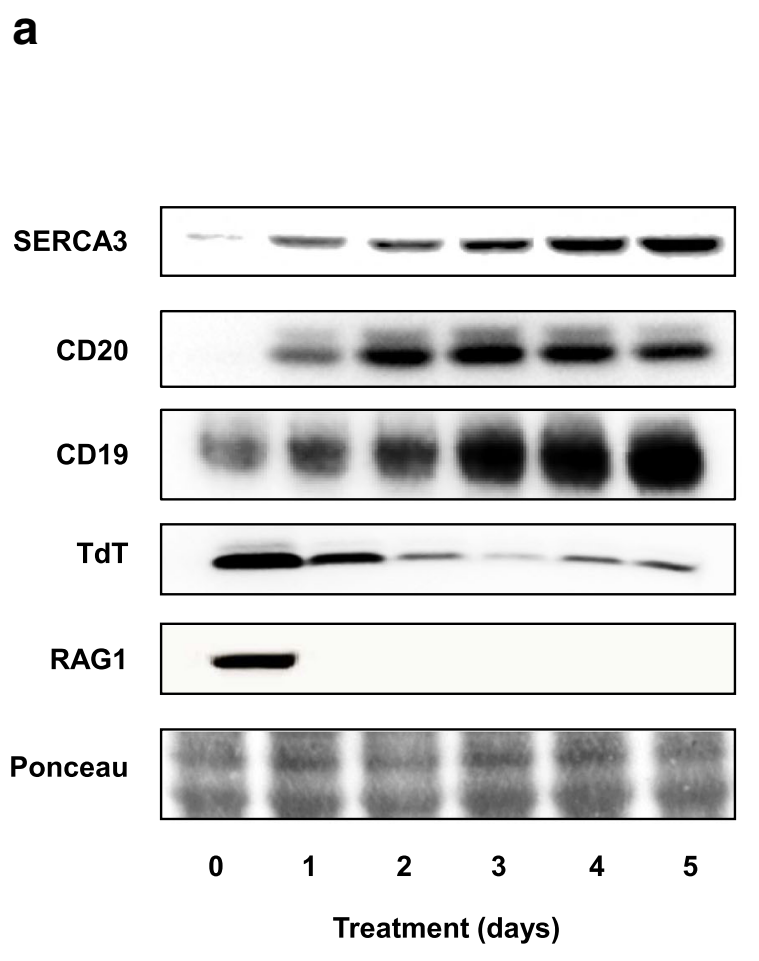

\section{b}
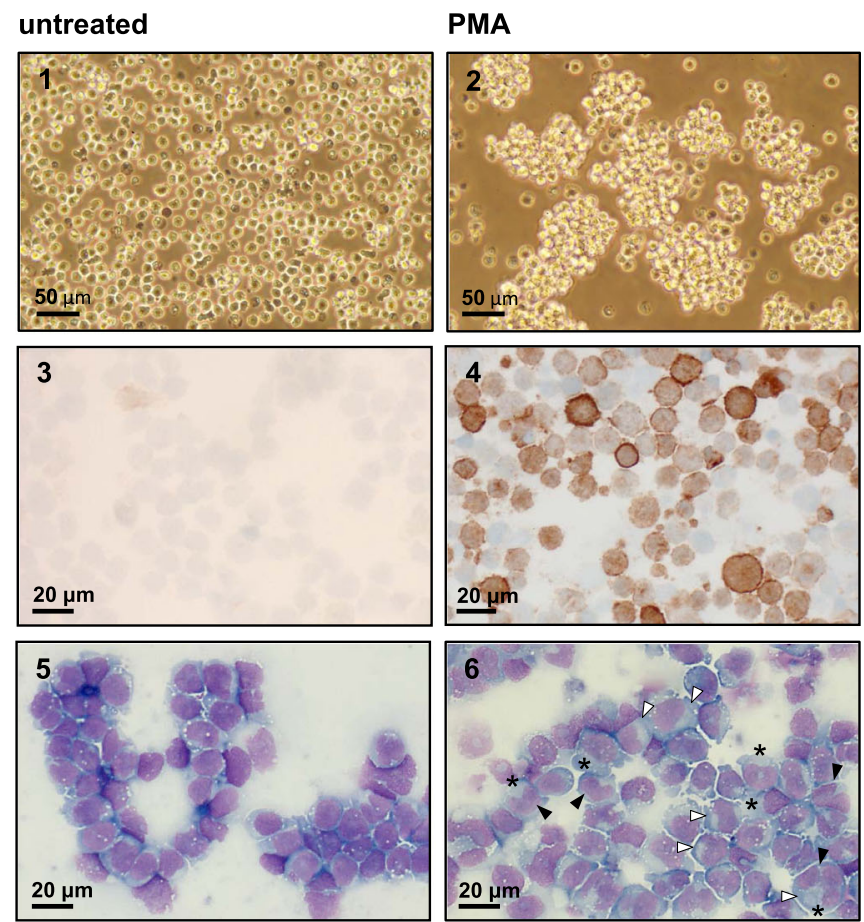

Fig. 4 Induction of differentiation by PMA in Kasumi-2 cells. a: Cells were treated with $10^{-7}$ M PMA during 5 days and the expression of SERCA3, CD20, TdT, RAG-1 and CD19 was determined by Western blotting daily. Whereas CD19 is expressed in untreated cells, induction of SERCA3 and CD20 expression by PMA treatment was accompanied by the down-regulation of TdT and RAG-1. b, photographs 1 and 2: PMA treatments led to the formation of multicellular aggregates. Whereas untreated cells (Photograph 1) grew predominantly as single cells in suspension, PMA treated cells ( $10^{-8} \mathrm{M}$, 4 days) formed floating multicellular aggregates (Photograph 2; original magnification: 20x; bars: 50 um). b, photographs 3 and 4: Induction of CD20 expression in PMA-treated Kasumi-2 cells detected by immunohistochemistry. Cytological smears of untreated (Photograph 3) and PMA-treated cells ( $10^{-8} \mathrm{M}, 4$ days, photograph 4) were stained for CD20 expression by immunohistochemistry as described in Methods. Brown staining indicates CD20 expression (original magnification: 40x; bars: $20 \mu \mathrm{m}$ ). b, photographs 5 and 6: May-Grünwald-Giemsa staining of cytological smears of untreated (photograph 5) and PMA-treated cells ( $10^{-8} \mathrm{M}, 4$ days, photograph 6). PMA treatment led greater cell size, decreased nucleocytoplasmic ratio (asterisks) and the frequent formation of bilobed, often excentral nuclei (black arrowheads) with a paranuclear halo (white arrowheads; original magnification: 40x; bars: $20 \mu \mathrm{m}$ ) 
shown in Fig. 5, treatment of cells with $10^{-8} \mathrm{M}$ PMA for 4 days led to markedly enhanced expression of the CD19, CD20, CD22 and FMC7 differentiation markers, whereas the expression of markers of immaturity such as CD10 or TdT was decreased. PMA treatment, however, did not lead to $\mathrm{k}$ or $\lambda$ chain or IgM expression (not shown), indicating however, that the cells do not attain a mature B cell phenotype.

\section{Induction of SERCA3 expression is PKC-dependent}

The induction of SERCA3 expression by PMA was investigated in the presence or absence of pharmacological inhibitors of PKC. As shown in Fig. 6, when cells were preincubated with Gö 6983 or with GF 109203X for $30 \mathrm{~min}$, the consecutive addition of $10^{-8}$ M PMA (a maximally active concentration) was without effect on SERCA expression, indicating the involvement of conventional or novel-type PKC activity in PMA-induced SERCA expression.

\section{Comparison of SERCA3 expression in precursor B and mature $B$ cell lines}

In order to further correlate SERCA3 expression with the state of differentiation of neoplastic B cells, SERCA3 expression of precursor $\mathrm{B}$ cell lines was compared to that of an extensive set of randomly selected mature $B$ cell lines (see Additional file 1: Table S1). As shown in Fig. 7, SERCA3 expression in all individual precursor B cell lines was weaker than in any of the investigated mature cell lines. A highly significant, on average 15-fold higher SERCA3 expression could be observed in mature $\mathrm{B}$ cell lines when compared with the average expression in the precursor B cell group.

\section{SERCA activity is involved in the control of cell differentiation}

The role of SERCA function in the differentiation of leukemic B cells was investigated using thapsigargin, a high affinity, selective inhibitor of SERCA-dependent calcium transport [40]. As shown in Fig. 8, Treatment of

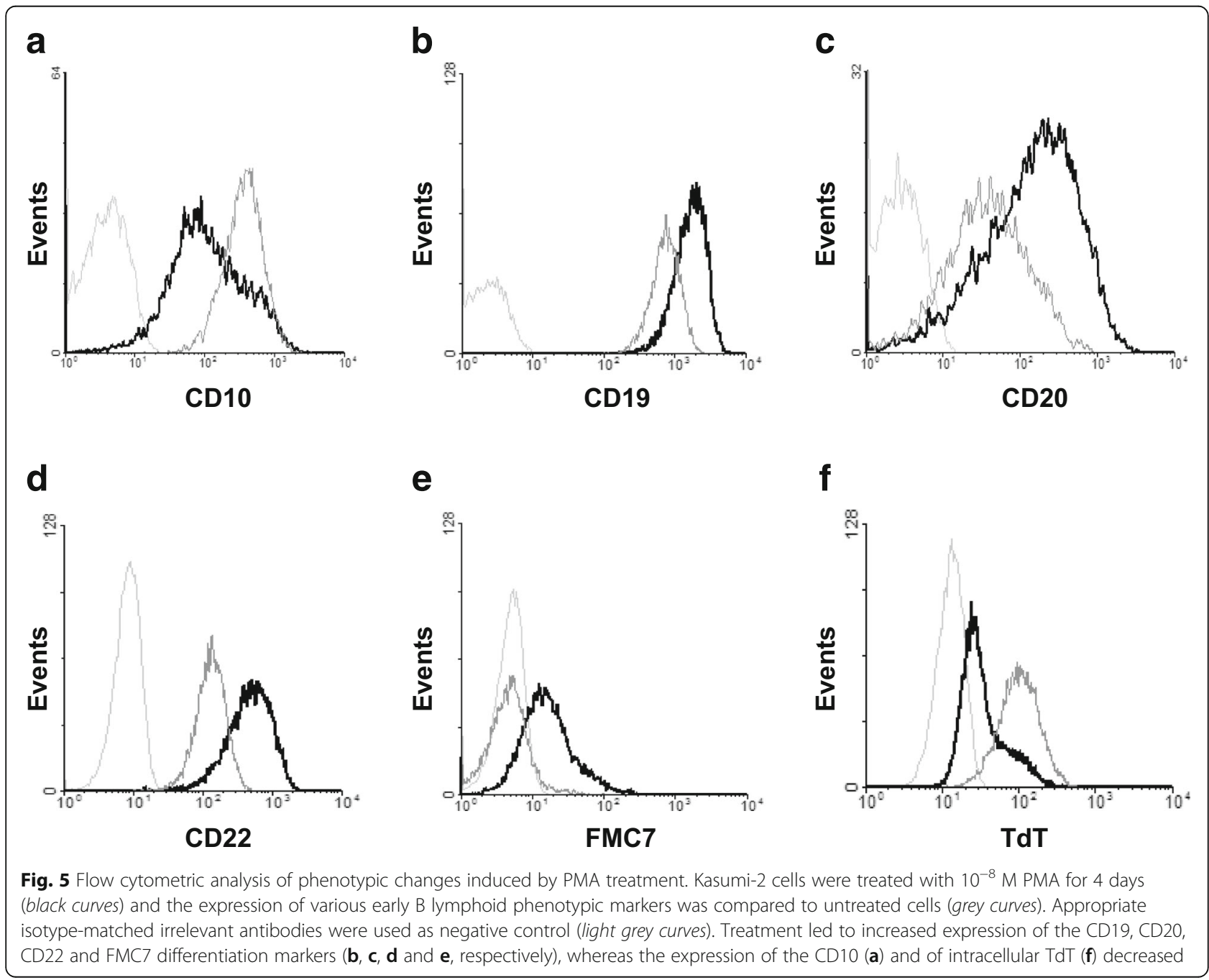




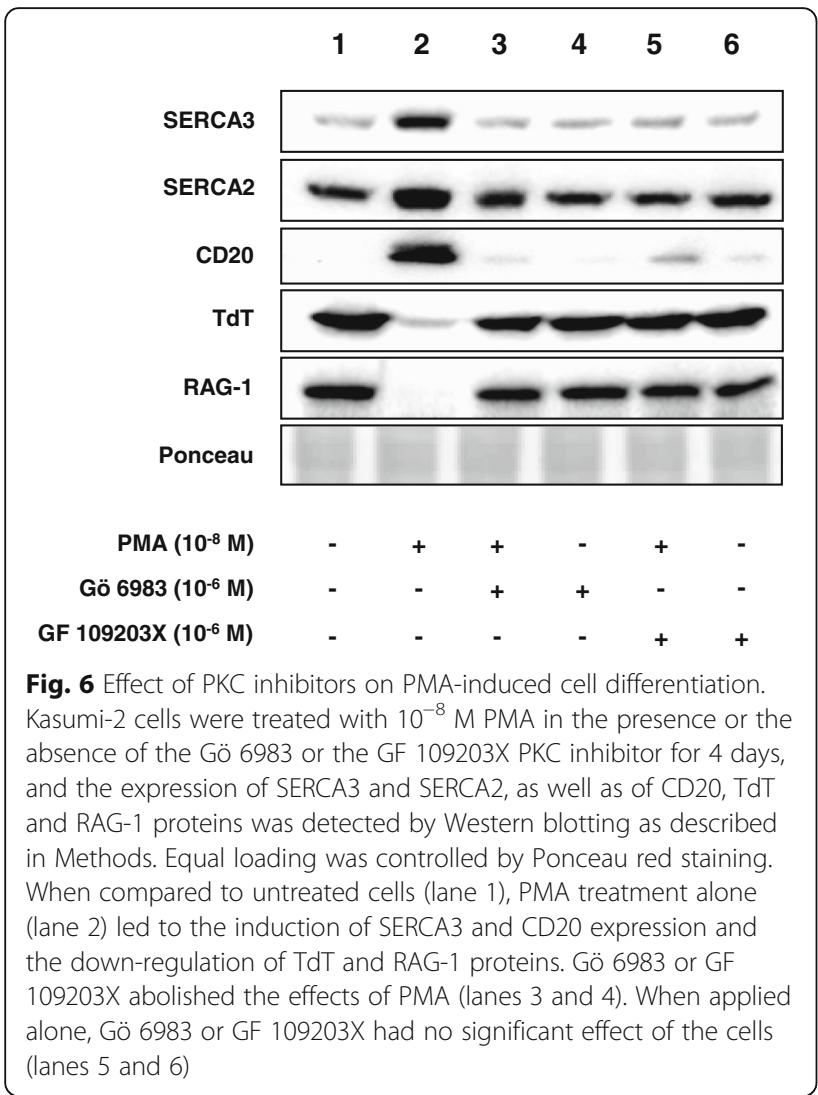

Kasumi-2 cells with sub-nanomolar concentrations of thapsigargin completely abolished the induction of CD20 expression induced by PMA (Fig. 8a). Inhibition of PMA-induced CD20 expression by thapsigargin was observed in the absence of significant toxicity during the experiments (Fig. 8b), although higher thapsigargin concentrations were, in accordance with the literature [41], toxic. Similar observations were made with cyclopiazonic acid or 2,5-di-tert-butyl-1,4-benzohydroquinone, two other, structurally unrelated SERCA inhibitors as well (data not shown).

\section{Discussion}

We show for the first time that ER calcium pump expression is remodeled during early B lymphoid differentiation. Activation of PKC-dependent signaling with a phorbol ester-type DAG analog in the Kasumi-2 B ALL cell line, blocked at the precursor B stage, led to the induction of differentiation of the cells as detected by the loss or decrease of expression of CD10, TdT and RAG-1 and the induction of expression of the CD19, CD20, CD22 and FMC7 markers, as well as morphological changes. This process was accompanied by a marked induction of the expression of SERCA3 mRNA and protein, whereas the expression of SERCA2 was only moderately increased. SERCA3 induction was observed on the protein, as well as the mRNA level. Whereas SERCA3 protein induction reached a plateau, mRNA induction followed a biphasic pattern. This may be due the dynamic interplay of various regulatory mechanisms, these, however, remain to be identified.

The induction of SERCA3 expression upon PMA treatment was observed in all B ALL cell lines investigated that carry the $t(1 ; 19)$ translocation giving rise to the E2A-PBX1 fusion oncoprotein, whereas in cell lines that belong to other molecular types such as $t(9 ; 22)$, $t(11 ; 19)$ or $t(12 ; 21)$ that express the BCR-ABL, MLLENL or TEL-AML1 fusion oncoproteins, respectively, we observed that PMA treatment in the same conditions led to rapid cell death. This suggests that the E2A-PBX1 expressing B ALL may constitute a molecular subtype in which differentiation blockage can be overcome by PKC activation. This may help to better understand the molecular mechanisms responsible for the inhibition of differentiation of this type of leukemia, should be investigated on primary leukemic cells ex vivo, and may be of potential importance for the development of targeted therapies in the future.

In addition to activating protein kinase $\mathrm{C}$ (PKC) isoenzymes belonging to the conventional and novel subfamilies, PMA can bind to and activate other DAG-binding proteins [42-47], including the RasGRP1 guanine nucleotide exchange factor [48]. However, the inhibition of the effects of PMA on the expression of SERCA3 and of other differentiation markers by PKC inhibitors indicates that PKC activation is the essential initiating step of the pharmacologically induced differentiation process that leads to the induction of SERCA3 expression in E2APBX1-expressing $t(1 ; 19)$ precursor B ALL cells. The precise identification of the PKC isoforms involved will require further studies such as, for example, PKC isoform-specific knock-down or gene invalidation.

Strong SERCA3 expression has already been observed in normal mature resting B lymphocytes in situ in lymph node follicles [20], and an approximately fivefold increase of SERCA3 mRNA levels can be seen in the ImmGen database [49] when pro- and pre-B cell populations and newly formed B cells are compared (see Additional file 2: Figure S1 and and Additional file 3 Figure: S2 for the expression profile of SERCA3, SERCA2 and various established differentiation markers, respectively), suggesting that enhanced SERCA3 expression occurs during normal B lymphocyte differentiation. The investigation of the cross-talk between key B cell differentiation regulatory mechanisms and SERCA function during in vitro differentiation of normal B lymphocyte precursors will help to better understand the underlying regulatory mechanisms, as well as the role of ER calcium homeostasis in B lymphocyte maturation. 


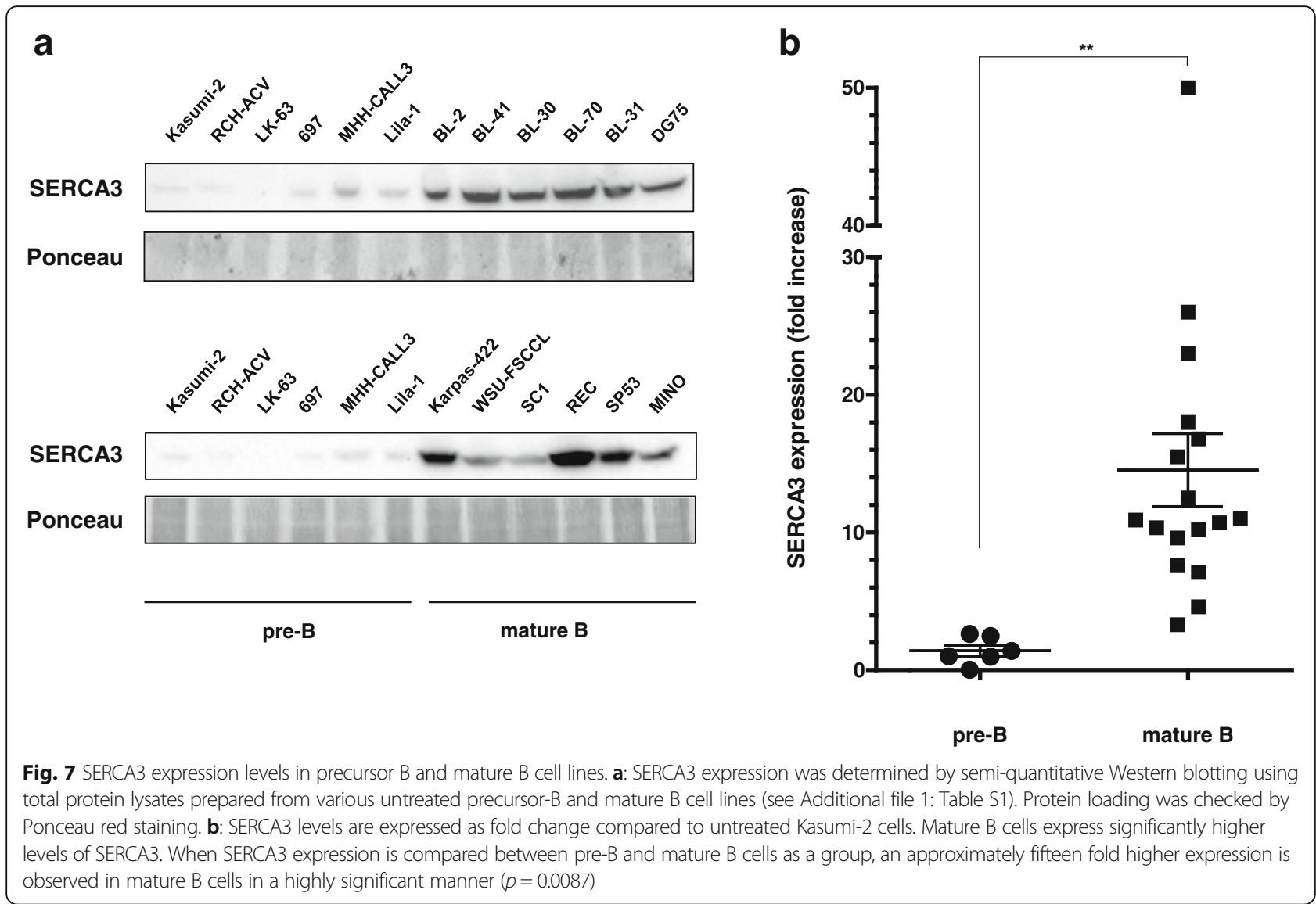

Our work indicates that E2A-PBX1-transformed BALL cells can be induced to differentiate by PKC activation, and that induction of SERCA3 mRNA and protein expression is induced during this process. A sharp contrast could also be observed when SERCA3 expression of precursor B ALL cell lines was compared to a large set of cell lines encompassing a wide range of mature $B$ neoplasia types. Despite variation among different cell lines, SERCA3 expression was several-fold higher in mature cells when compared to precursor B cell lines. This indicates that systematic differences exist in terms of SERCA3 expression between immature and mature B lymphoid malignancies, and that SERCA3 may therefore constitute a useful new phenotypic marker for the study of B lymphoid differentiation and leukemia/lymphoma phenotype analysis.

Calcium signaling plays an important role in the activation of mature $B$ cells $[5,50]$. Antigen binding to the $\mathrm{BCR}$ leads to phospholipase-C activation, IP3 production and calcium release from IP3-sensitive intracellular pools [50-52]. The calcium affinity of SERCA3 is inferior when compared to the simultaneously expressed SERCA2 isoform $[21 ; 23]$, and SERCA3 has been shown to be preferentially associated with the IP3-sensitive intracellular calcium compartment in platelets [53]. It is therefore tempting to hypothesize that the induction of SERCA3 expression during the maturation of B cell precursors is part of the differentiation program, whereby the cells acquire an intracellular calcium storage compartment poised to mount larger calcium release signals upon BCR activation.

When cell differentiation was conducted in the presence of sub-nanomolar, non-toxic concentrations of thapsigargin, a high affinity pan-SERCA inhibitor, induction of CD20 expression by PMA was completely suppressed. Similar results were obtained by using cyclopiazonic acid and 2,5-di(tert)butyl-1,4-benzohidroquinone, two other, structurally unrelated SERCA inhibitors as well. These observations show, that SERCA function is required for differentiation to proceed, and that the perturbation of SERCA activity can interfere with this process. The identification of the relative contribution of SERCA3 and SERCA2 to cell differentiation will require further studies based on the direct, selective modulation of SERCA3 and SERCA2 levels. This can be accomplished by isoenzymespecific transgene overexpression, gene invalidation or knock-down techniques. When applied in conjunction with genetically engineered fluorescent calcium indicators 

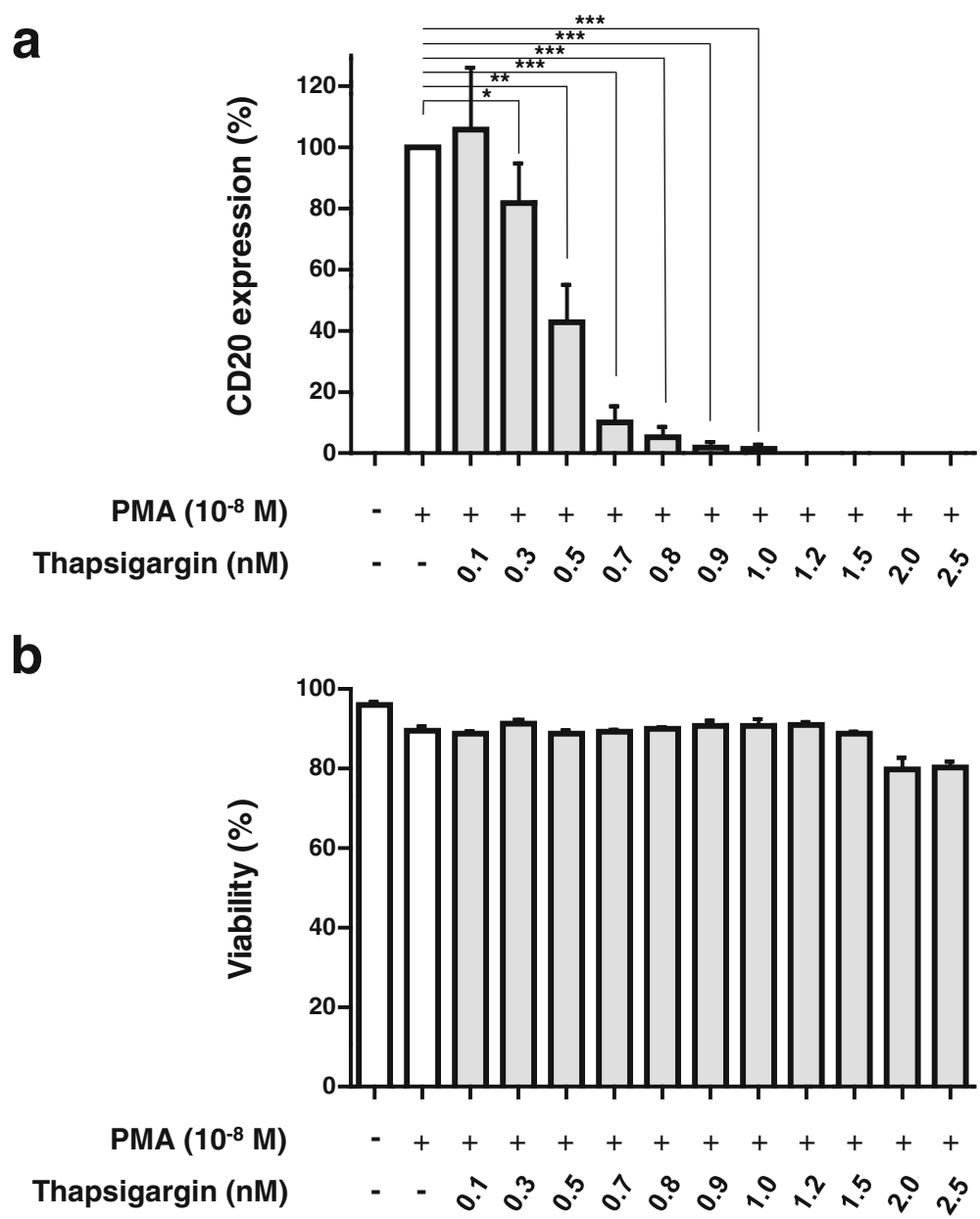

Fig. 8 Effect of SERCA inhibition on PMA-induced expression of CD20 in Kasumi-2 cells. a: Cells were incubated with $10^{-8}$ M PMA in the absence (empty column) or in the presence (gray columns) of various thapsigargin concentrations in the sub- to low nanomolar concentration range for 4 days, and CD20 expression was measured by semi-quantitative Western blotting. Thapsigargin treatment led to a concentration-dependent inhibition of PMA-induced CD20 expression. Although higher concentrations of thapsigargin led to cell death, no major cytotoxic effects were observed at the concentrations used in these experiments (b)

(GECIs) targeted to the ER lumen or the cytosol, these studies will allow to better understand the functional cross-talk that occurs between ER calcium homeostasis and the control of cell differentiation and its defects in leukemia.

\section{Conclusions}

In summary, this work shows, for the first time that SERCA3 expression is induced during early B cell differentiation, inhibition of SERCA3 expression and the differentiation block in pre-B ALL cells can be overcome by PKC activation, and that unperturbed SERCA function is required for cell differentiation. In addition, these data indicate that SERCA3 may serve as a useful new phenotypic marker for the study of B cell differentiation and its defects in leukemia.

\section{Additional files}

Additional file 1: Table S1. Name, hematological origin and molecular type of the cell lines used in this study. (DOCX $18 \mathrm{~kb}$ )

Additional file 2: Figure S1. Expression profile of SERCA3 and SERCA2 mRNA in key normal early B cell populations in the mouse, adapted from the Immunological Genome project transcriptomic database. (PPTX 1190 kb)

Additional file 3: Figure S2. Expression profile of CD19, CD20 CD22, CD34, TdT and of $\beta$-actin in key normal early B cell populations in the mouse, adapted from the Immunological Genome project transcriptomic database. (PPTX $566 \mathrm{~kb}$ )

\section{Abbreviations}

ALL: acute lymphoblastic leukemia; ATCC: American Type Culture Collection; BCR: B cell antigen receptor; DAB: 3,3'-diaminobenzidine; DAG: diacyl-glycerol; DMSO: dimethyl-sulfoxide; DSMZ: Deutsche Sammlung von Mikroorganismen und Zellkulturen GmbH; E2A: transcription factor 3 (TCF3); EBV: Epstein-Barr virus; ECACC: European Collection of Cell Cultures; ER: endoplasmic reticulum; GAPDH: glyceraldehyde 3-phosphate dehydrogenase; IP3: D-myo-inositol-1,4,5trisphosphate; NFAT: Nuclear factor of activated T cells; NF-KB: Nuclear Factor 
kappa-light-chain-enhancer of activated B cells; PBX1: pre-B-cell leukemia transcription factor 1; PKC: protein kinase C; PLC- $\gamma$ : phospholipase C-gamma; PMA: phorbol-12-myristate-13-acetate; RAG-1: recombination-activating gene 1; RT-PCR: reverse transcription polymerase chain reaction; SDS PAGE: Sodium dodecyl-sulfate polyacrylamide gel electrophoresis; SERCA: sarco/endoplasmic reticulum calcium ATPase; TCA: trichloroacetic acid; TdT: Terminal deoxynucleotidyl transferase

\section{Acknowledgements}

This work benefitted from data assembled by the ImmGen consortium. We thank Prof. N. Crawford (Royal College of Surgeons of England, London, UK) for the PLIM430 hybridoma. This work is dedicated to the memory of Mrs Ould-Houcine Tassadit.

\section{Funding}

This work was supported by Fondation ARC pour la Recherche sur le Cancer, France.

\section{Availability of data and materials}

Not applicable.

\section{Authors' contributions}

$B P, L A G$ and AA designed the study, gathered and analyzed the majority of the data. JPB, FBM, RL and HR gathered and analyzed data regarding morphology, flow cytometry and RQ-PCR, respectively. ES, AB and HGD contributed vital reagents. AE and NVB contributed to experimental design and data analysis. BP edited the manuscript with the help of all authors, who read and approved the final manuscript.

\section{Competing interests}

The authors declare that they have no competing interests.

\section{Consent for publication}

Not applicable.

\section{Ethics approval and consent to participate}

Not applicable.

\section{Publisher's note}

Springer Nature remains neutral with regard to jurisdictional claims in published maps and institutional affiliations.

\section{Author details}

'Institut National de la Santé et de la Recherche Médicale, U978 Bobigny, France. ${ }^{2}$ Université Paris-13, PRES Sorbonne Paris-Cité, 74, rue Marcel Cachin 93017, Bobigny, France. ${ }^{3}$ Département de Virologie, Institut Pasteur, Paris, France. ${ }^{4}$ Service d'Anatomie et Cytologie Pathologiques, Hôpital Lariboisière, Paris, France. ${ }^{5}$ Service d'Hématologie Biologique, Hôpitaux Universitaires Paris Seine-Saint-Denis, AP-HP, Hôpital Avicenne, Bobigny, France. ${ }^{6}$ mmunology Research Centre, St Vincent's Hospital, Melbourne, VIC, Australia. ${ }^{7}$ Department of Medicine, University of Queensland, Queensland, Australia. ${ }^{8}$ Deutsche Sammlung von Mikroorganismen und Zellkulturen GmbH, Brauschweig, Germany. ${ }^{9}$ Second Institute of Pathology, Semmelweis University Medical School, Budapest, Hungary. ${ }^{10}$ U978 Inserm, UFR SMBH, Université Paris-13, 74, rue Marcel Cachin, 93017 Bobigny, France.

Received: 24 January 2017 Accepted: 18 June 2017 Published online: 26 June 2017

\section{References}

1. Baba Y, Kurosaki T. Impact of $\mathrm{Ca}^{2+}$ signaling on B cell function. Trends Immunol. 2011;32:589-94.

2. Feske S, Skolnik EY, Prakriya M. Ion channels and transporters in lymphocyte function and immunity. Nat Rev Immunol. 2012;12:532-47.

3. Marshall AJ, Niiro H, Yun TJ, Clark EA. Regulation of B-cell activation and differentiation by the phosphatidylinositol 3-kinase and phospholipase Cgamma pathway. Immunol Rev. 2000;176:30-46.

4. Vig M, Kinet JP. Calcium signaling in immune cells. Nat Immunol. 2009;10: 21-7.

5. Oh-hora M, Rao A. Calcium signaling in lymphocytes. Curr Opin Immunol. 2008;20:250-8
6. Streb H, Irvine RF, Berridge MJ, Schulz I. Release of $\mathrm{Ca}^{2+}$ from a nonmitochondrial intracellular store in pancreatic acinar cells by inositol1,4,5-trisphosphate. Nature. 1983;306:67-9.

7. Zhang SL, Yu Y, Roos J, Kozak JA, Deerinck TJ, Ellisman MH, Stauderman KA, Cahalan MD. STIM1 is a $\mathrm{Ca}^{2+}$ sensor that activates CRAC channels and migrates from the $\mathrm{Ca}^{2+}$ store to the plasma membrane. Nature. 2005;437:902-5.

8. Prakriya M, Feske S, Gwack Y, Srikanth S, Rao A, Hogan PG. Orai1 is an essential pore subunit of the CRAC channel. Nature. 2006;443:230-3.

9. Park J, Yaseen NR, Hogan PG, Rao A, Sharma S. Phosphorylation of the transcription factor NFATp inhibits its DNA binding activity in cyclosporin Atreated human B and T cells. J Biol Chem. 1995;270:20653-9.

10. Nelsen B, Hellman L, Sen R. The NF-kappa B-binding site mediates phorbol ester-inducible transcription in nonlymphoid cells. Mol Cell Biol. 1988;8:3526-31.

11. Periasamy M, Kalyanasundaram A. SERCA pump isoforms: their role in calcium transport and disease. Muscle Nerve. 2007:35:430-42.

12. Strehler EE, Treiman M. Calcium pumps of plasma membrane and cell interior. Curr Mol Med. 2004:4:323-35.

13. Wuytack F, Raeymaekers L, Missiaen L. Molecular physiology of the SERCA and SPCA pumps. Cell Calcium. 2002;32:279-305.

14. MacLennan DH, Brandl CJ, Korczak B, Green NM. Amino-acid sequence of a $\mathrm{Ca}^{2+}+\mathrm{Mg}^{2+}$-dependent ATPase from rabbit muscle sarcoplasmic reticulum, deduced from its complementary DNA sequence. Nature. 1985;316:696-700.

15. Bian JH, Ghosh TK, Wang JC, Gill D. Identification of intracellular calcium pools. Selective modification by thapsigargin. J Biol Chem. 1991;266: 8801-6.

16. Cheek TR, Thastrup O. Internal $\mathrm{Ca}^{2+}$ mobilization and secretion in bovine adrenal chromaffin cells. Cell Calcium. 1989;10:213-21.

17. Visegrády A, Lakos Z, Czimbalek L, Somogyi B. Stimulus-dependent control of inositol 1,4,5-trisphosphate-induced $\mathrm{Ca}^{2+}$ oscillation frequency by the endoplasmic reticulum Ca ${ }^{2+}$-ATPase. Biophys J. 2001;81:1398-405.

18. Dolmetsch RE, Xu K, Lewis RS. Calcium oscillations increase the efficiency and specificity of gene expression. Nature. 1998;392:933-6.

19. Papp B, Enyedi Á, Pászty K, Kovács T, Sarkadi B, Gárdos G, Magnier C, Wuytack F, Enouf J. Simultaneous presence of two distinct endoplasmic-reticulum-type calcium-pump isoforms in human cells. Characterization by radioimmunoblotting and inhibition by 2,5-di-(t-butyl)-1,4-benzohydroquinone. Biochem J. 1992;288(Pt 1):297-302.

20. Dellis O, Arbabian A, Brouland J-P, Kovács T, Rowe M, Chomienne C, Joab I, Papp B. Modulation of B-cell endoplasmic reticulum calcium homeostasis by Epstein-Barr virus latent membrane protein-1. Mol Cancer. 2009:8:59.

21. Chandrasekera PC, Kargacin ME, Deans JP, Lytton J. Determination of apparent calcium affinity for endogenously expressed human sarco(endo)plasmic reticulum calcium-ATPase isoform SERCA3. Am J Physiol Cell Physiol. 2009;296: C1105-14.

22. Vandecaetsbeek I, Vangheluwe P, Raeymaekers L, Wuytack F, Vanoevelen J. The $\mathrm{Ca}^{2+}$ pumps of the endoplasmic reticulum and Golgi apparatus. Cold Spring Harb Perspect Biol. 2011:1:3(5).

23. Vandecaetsbeek I, Trekels M, De Maeyer M, Ceulemans H, Lescrinier E, Raeymaekers L, Wuytack F, Vangheluwe P. Structural basis for the high Ca2+ affinity of the ubiquitous SERCA2b Ca ${ }^{2+}$ pump. Proc Natl Acad Sci U S A. 2009:106:18533-8.

24. Vangheluwe $P$, Sepulveda MR, Missiaen L, Raeymaekers L, Wuytack F, Vanoevelen J. Intracellular $\mathrm{Ca}^{2+}$ - and $\mathrm{Mn}^{2+}$-transport ATPases. Chem Rev. 2009; 109:4733-59.

25. Arbabian A, Brouland J-P, Gélébart P, Kovács T, Bobe R, Enouf J, Papp B. Endoplasmic reticulum calcium pumps and cancer. Biofactors. 2011;37: 139-49.

26. Papp B, Brouland J-P, Arbabian A, Gélébart P, Kovács T, Bobe R, Enouf J, Varin-Blank N, Apáti Á. Endoplasmic reticulum calcium pumps and cancer cell differentiation. Biomolecules. 2012:2:165-86.

27. Herzog S, Reth $\mathrm{MH}$, Jumaa $\mathrm{H}$. Regulation of B-cell proliferation and differentiation by pre-B-cell receptor signalling. Nat Rev Immunol. 2009; 9:195-205.

28. Ikawa T. Genetic and epigenetic control of early lymphocyte development. Curr Top Microbiol Immunol. 2014;381:1-20.

29. Rothenberg EV. Transcriptional control of early T and B cell developmental choices. Annu Rev Immunol. 2014;32:283-321.

30. Inaba H, Greaves M, Mullighan CG. Acute lymphoblastic leukaemia. Lancet. 2013;381:1943-55.

31. Mullighan CG. Molecular genetics of B-precursor acute lymphoblastic leukemia. J Clin Invest. 2012;122:3407-15. 
32. Castagna M, Takai Y, Kaibuchi K, Sano K, Kikkawa U, Nishizuka Y. Direct activation of calcium-activated, phospholipid-dependent protein kinase by tumor-promoting phorbol esters. J Biol Chem. 1982;257:7847-51.

33. Lacabaratz-Porret C, Launay S, Corvazier E, Bredoux R, Papp B, Enouf J. Biogenesis of endoplasmic reticulum proteins involved in $\mathrm{Ca}^{2+}$ signalling during megakaryocytic differentiation: an in vitro study. Biochem J. 2000; 350(Pt 3):723-34

34. Launay $S$, Giannì M, Kovács T, Bredoux R, Bruel A, Gélébart P, Zassadowski F, Chomienne C, Enouf J, Papp B. Lineage-specific modulation of calcium pump expression during myeloid differentiation. Blood. 1999;93:4395-405.

35. Arbabian A, Brouland J-P, Apáti Á, Paszty K, Hegedüs L, Enyedi A, Chomienne C, Papp B. Modulation of endoplasmic reticulum calcium pump expression during lung cancer cell differentiation. FEBS J. 2013;280:5408-18.

36. Le Roy C, Varin-Blank N, Ajchenbaum-Cymbalista F, Letestu R. Flow cytometry APC-tandem dyes are degraded through a cell-dependent mechanism. Cytometry A. 2009;75:882-90.

37. Craig FE, Foon KA. Flow cytometric immunophenotyping for hematologic neoplasms. Blood. 2008;111:3941-67.

38. Livak KJ, Schmittgen TD. Analysis of relative gene expression data using real-time quantitative PCR and the 2(-Delta Delta C(T)) Method. Methods. 2001;25:402-8.

39. Salvaris E, Novotny JR, Welch K, Campbell L, Boyd AW. Characterization of two novel pre-B-cell lines (LK63 and LiLa-1): potential models of pre-B-cell differentiation. Leuk Res. 1992;16:655-63.

40. Thastrup O, Cullen PJ, Drobak BK, Hanley MR, Dawson AP. Thapsigargin, a tumor promoter, discharges intracellular $\mathrm{Ca}^{2+}$ stores by specific inhibition of the endoplasmic reticulum $\mathrm{Ca}^{2+}$-ATPase. Proc Natl Acad Sci U S A. 1990;87: 2466-70.

41. Andersen TB, Lopez CQ, Manczak T, Martinez K, Simonsen HT. Thapsigarginfrom Thapsia L. to mipsagargin. Molecules. 2015;20:6113-27.

42. Gaggioli C, Hooper S, Hidalgo-Carcedo C, Grosse R, Marshall JF, Harrington K, Sahai E. Fibroblast-led collective invasion of carcinoma cells with differing roles for RhoGTPases in leading and following cells. Nat Cell Biol. 2007;9: 1392-400.

43. Bruinsma SP, Baranski TJ. Beta2-chimaerin in cancer signaling: connecting cell adhesion and MAP kinase activation. Cell Cycle. 2007;6:2440-4.

44. Wang QJ. PKD at the crossroads of DAG and PKC signaling. Trends Pharmacol Sci. 2006;27:317-23.

45. Topham MK. Signaling roles of diacylglycerol kinases. J Cell Biochem. 2006; 97:474-84.

46. Stone JC. Regulation of Ras in lymphocytes: get a GRP. Biochem Soc Trans. 2006;34:858-61.

47. Silinsky EM, Searl TJ. Phorbol esters and neurotransmitter release: more than just protein kinase C? Br J Pharmacol. 2003;138:1191-201.

48. Ebinu JO, Bottorff DA, Chan EY, Stang SL, Dunn RJ, Stone JC. RasGRP, a Ras guanyl nucleotide- releasing protein with calcium- and diacylglycerolbinding motifs. Science. 1998;280:1082-6.

49. Heng TS, Painter MW. The Immunological Genome Project: networks of gene expression in immune cells. Nat Immunol. 2008;9:1091-4.

50. Cantrell D. Signaling in lymphocyte activation. Cold Spring Harb Perspect Biol. 2015;1:7(6)

51. Berridge MJ. Inositol trisphosphate and calcium signalling mechanisms. Biochim Biophys Acta. 2009;1793:933-40.

52. Baba Y, Matsumoto M, Kurosaki T. Calcium signaling in B cells: regulation of cytosolic $\mathrm{Ca}^{2+}$ increase and its sensor molecules, STIM1 and STIM2. Mol Immunol. 2014;62:339-43.

53. Papp B, Pászty K, Kovács T, Sarkadi B, Gárdos G, Enouf J, Enyedi A Characterization of the inositol trisphosphate-sensitive and insensitive calcium stores by selective inhibition of the endoplasmic reticulum-type calcium pump isoforms in isolated platelet membrane vesicles. Cell Calcium. 1993:14:531-8

\section{Submit your next manuscript to BioMed Central and we will help you at every step:}

- We accept pre-submission inquiries

- Our selector tool helps you to find the most relevant journal

- We provide round the clock customer support

- Convenient online submission

- Thorough peer review

- Inclusion in PubMed and all major indexing services

- Maximum visibility for your research

Submit your manuscript at www.biomedcentral.com/submit
) Biomed Central 